\title{
ERNE observations of energetic particles associated with Earth-directed coronal mass ejections in April and May, 1997
}

\author{
A. Anttila, T. Sahla \\ Space Research Laboratory, University of Turku, Physics Department, Vesilinnantie 5, FIN-20014 Turku University, Finland
}

Received: 19 July 1999 / Revised: 3 July 2000 / Accepted: 4 July 2000

\begin{abstract}
Two Earth-directed coronal mass ejections (CMEs), which were most effective in energetic $(\sim 1-$ $50 \mathrm{MeV}$ ) particle acceleration during the first 18 months since the Solar and Heliospheric Observatory (SOHO) launch, occurred on April 7 and May 12, 1997. In the analysis of these events we have deconvoluted the injection spectrum of energetic protons by using the method described by Anttila et al. In order to apply the method developed earlier for data of a rotating satellite (Geostationary Operational Environmental Satellites, GOES), we first had to develop a method to calculate the omnidirectional energetic particle intensities from the observations of Energetic and Relativistic Nuclei and Electrons (ERNE), which is an energetic particle detector onboard the three-axis stabilized SOHO spacecraft. The omnidirectional intensities are calculated by fitting an exponential pitch angle distribution from directional information of energetic protons observed by ERNE. The results of the analysis show that, compared to a much faster and more intensive CMEs observed during the previous solar maximum, the acceleration efficiency decreases fast when the shock propagates outward from the Sun. The particles injected at distances $<0.5 \mathrm{AU}$ from the Sun dominate the particle flux during the whole period, when the shock propagates to the site of the spacecraft. The main portion of particles injected by the shock during its propagation further outward from the Sun are trapped around the shock, and are seen as an intensity increase at the time of the shock passage.
\end{abstract}

Key words: Interplanetary physics (interplanetary shocks) - Solar physics, astrophysics and astronomy (energetic particles; flares and mass ejections)

\section{Introduction}

CMEs are vast outbursts of solar material, during which typically $10^{9}-10^{10}$ tons of plasma is ejected into interplanetary (IP) space with speeds ranging from less than 50 to more than $2000 \mathrm{~km} / \mathrm{s}$ (e.g., Gosling, 1997). If a CME propagates sufficiently faster than the ambient solar wind, a shock is formed in front of it. During the first half of the year 1997, several Earth-directed CMEs, which were capable to accelerate particles up to $\mathrm{MeV}$ energies, occurred (for detailed analysis of these events, see the special issue of Geophys. Res. Lett., vol 25, (14), 1998). The events on April 7 and May 12 were the most intensive ones in energetic particle acceleration, being capable of accelerating particles up to energies above $50 \mathrm{MeV}$ (Torsti, et al., 1998).

The energetic particle acceleration during CMEs has been the subject of much debate. The old ideas, which proposed that flare accelerated particles are stored in the solar corona and are gradually diffused into interplanetary magnetic field lines connected to the observer, have been replaced. This is because elemental abundances and ionic charge states during gradual events are characteristic of solar corona and solar wind, and the often detected intensity increase of energetic particles during a passage of IP shock indicates that the particle population is accelerated by the shock (e.g., Gosling, 1993). Models, in which the CME-driven shock accelerates particles from solar wind, have also not been able to explain how particles can reach energies up to tens or hundreds of MeVs (Lim et al., 1995; Kallenrode, 1996). Also the charge states of energetic $\mathrm{Fe}$ ions are similar to coronal plasma, not solar wind (Boberg et al., 1996). A recent study by Klein et al. (1999) gives evidence that the initial pre-acceleration up to $\sim 1 \mathrm{MeV}$ close to the Sun suggested by Tan et al. (1989) and Anttila et al. (1998) takes place behind the CME.

We calculate the injection spectrum of solar energetic particles (SEP) from the observations of ERNE experiment onboard SOHO. The methods used in this study 
are a previously developed deconvolution method (Anttila et al., 1998) and a new method to calculate the omnidirectional intensities of SEPs from the intensity and anisotropy observations of a three-axis stabilized spacecraft.

\section{The instrument}

ERNE consists of two energetic particle telescopes, both of which detect energetic ions in the elemental range $\mathrm{Z}=1-30$. The energy range of protons for Low Energy Detector (LED) is $1.6-13 \mathrm{MeV}$ and for High Energy Detector (HED) 12-100 MeV. A complete description of ERNE is given by Torsti et al. (1995).

LED is a two-layer silicon detector. The upper layer consists of seven different circular windows, D11-D17, with a common lower layer D2. The central window D17 is parallel with D2 and the others are tilted by $16^{\circ}$ from it into directions separated by $60^{\circ}$ from the adjacent. The geometric factor of LED varies from 0.26 to $0.915 \mathrm{~cm}^{2}$ sr depending on the particle energy.

HED consists of five layers of silicon detectors (S1x, S1y, S2x, S2y and D1), and two scintillators (D2 and D3) which are surrounded by an anticoincidence shield (AC). The structure is shown in Fig. 1. The uppermost layer, S1x, consists of two parallel single-sided $33 \times 70$ $\mathrm{mm}^{2}$ position sensitive silicon strip detectors side by side, with a $4 \mathrm{~mm}$ inactive area in between. Thus the total area of $\mathrm{S} 1 \mathrm{x}$ is $70 \times 70 \mathrm{~mm}^{2}$, but it has a $4 \times 70 \mathrm{~mm}^{2}$ inactive region in the middle. The second layer, S1y, has the same structure as S1x, but the strip directions in the two layers are perpendicular. Together S1x and S1y measure the coordinates where the particle penetrates the S1 plane, with an accuracy of $1 \mathrm{~mm}$ (the width of one strip). S2x and S2y form an equal pair $31 \mathrm{~mm}$ lower, and thus the particle trajectory can be determined from the measurements of these four detector layers with an accuracy of $\sim 1^{\circ}$. This enables HED to show high accuracy in analysis of flux directionality. The geometric factor for HED is $36 \mathrm{~cm}^{2}$ sr for $14 \mathrm{MeV}$ protons. The view cone diminishes with the increase of

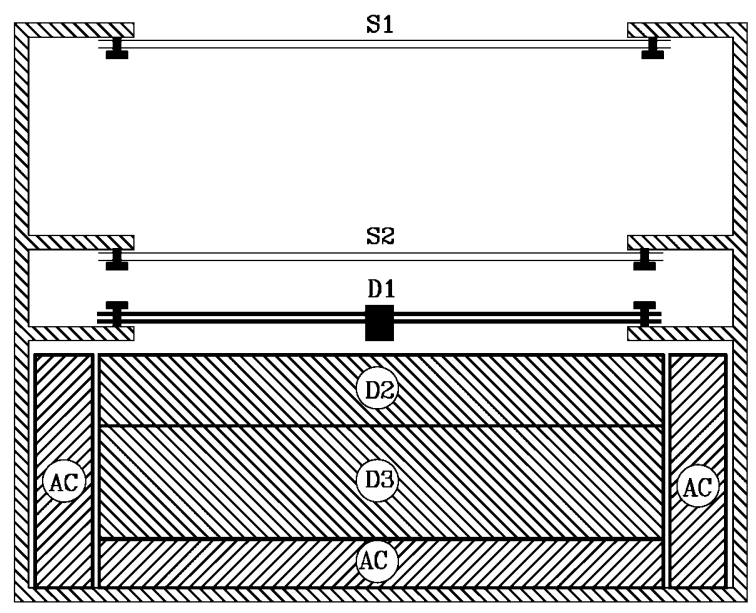

Fig. 1. The construction of ERNE/HED. For details see text the proton energy, and the geometric factor for $100 \mathrm{MeV}$ protons is $25 \mathrm{~cm}^{2} \mathrm{sr}$. The dependence of the geometric factor of HED on the energy spectrum and the three dimensional direction in the view cone has been compensated for in the analysis by determining the differential geometric acceptance as a function of particle direction and energy.

The directional information of magnetic field needed for this study is obtained from Magnetic Field Investigation (MFI) instrument onboard WIND spacecraft (http://lepmfi.gsfc.nasa.gov/mfi/windmfi.html). The solar wind speed data is obtained from Charge, Element and Isotope Analysis System (CELIAS/MTOF) Proton Monitor onboard SOHO (http://umtof.umd.edu/pm).

\section{The model}

The intensity within HED view cone is measured in pitch angle bins (the size of which varies between $5^{\circ}$ and $30^{\circ}$ depending on statistics) around the magnetic field direction acquired from WIND/MFI. As the ERNE view cone is a $120^{\circ}$ wide square pointed along the nominal direction of IMF, this method gives a 6-10 point pitch angle distribution. The spatial separation of the SOHO and WIND spacecraft is taken into account as a temporal difference by shifting the WIND/MFI measurement for the amount of time that it takes for the Parker magnetic field line in contact with WIND to move into contact with SOHO. The solar wind speed needed to determine the Parker field configuration is acquired from CELIAS measurement. The intensity of protons/ $\left(\mathrm{cm}^{2} \mathrm{sr} \mathrm{s} \mathrm{MeV}\right)$ as the function of the pitch angle under assumption of isotropic scattering is of the form

$I(\theta)=b_{0} \exp \left(b_{1} \cos (\theta)\right)$,

where $\mathrm{I}(\theta)$ is the intensity in protons $/\left(\mathrm{cm}^{2}\right.$ sr s MeV). Parameters $b_{0}$ and $b_{1}$ are determined by fitting the observed pitch angle distribution to this formula. The parameter $b_{0}$ is the intensity perpendicular to the magnetic field, and $b_{1}$ indicates the amount of anisotropy present in the flux. Thus the model is a simplification of the model presented by Beeck and Wibberenz (1986). The simplification is justified by Monte-Carlo simulations presented by Vainio (1998). The energy range for this fit was selected to be $14.1-35.5 \mathrm{MeV}$ as a compromise between reasonable statistics and an energy range narrow enough.

Figure 2 shows six samples of pitch angle distributions through the May 12, 1997 event. The solid lines are fits based on Eq. 1. It can easily be seen that during periods like May 12, 9:44-9:59 UT, our fitting method works well, but periods like May 12, 11:29-11:44 UT, hint at a pitch angle distribution, which is not of the form of Eq. 1. In the case of LED, we used the simulations of Lumme (1995) to determine effective geometric factors of LED windows as a function of angle of incidence.

The model for deconvolution of the spectrum in the source is the same as presented by Anttila et al. (1998). We adopted the following parametrization (Ellison and 

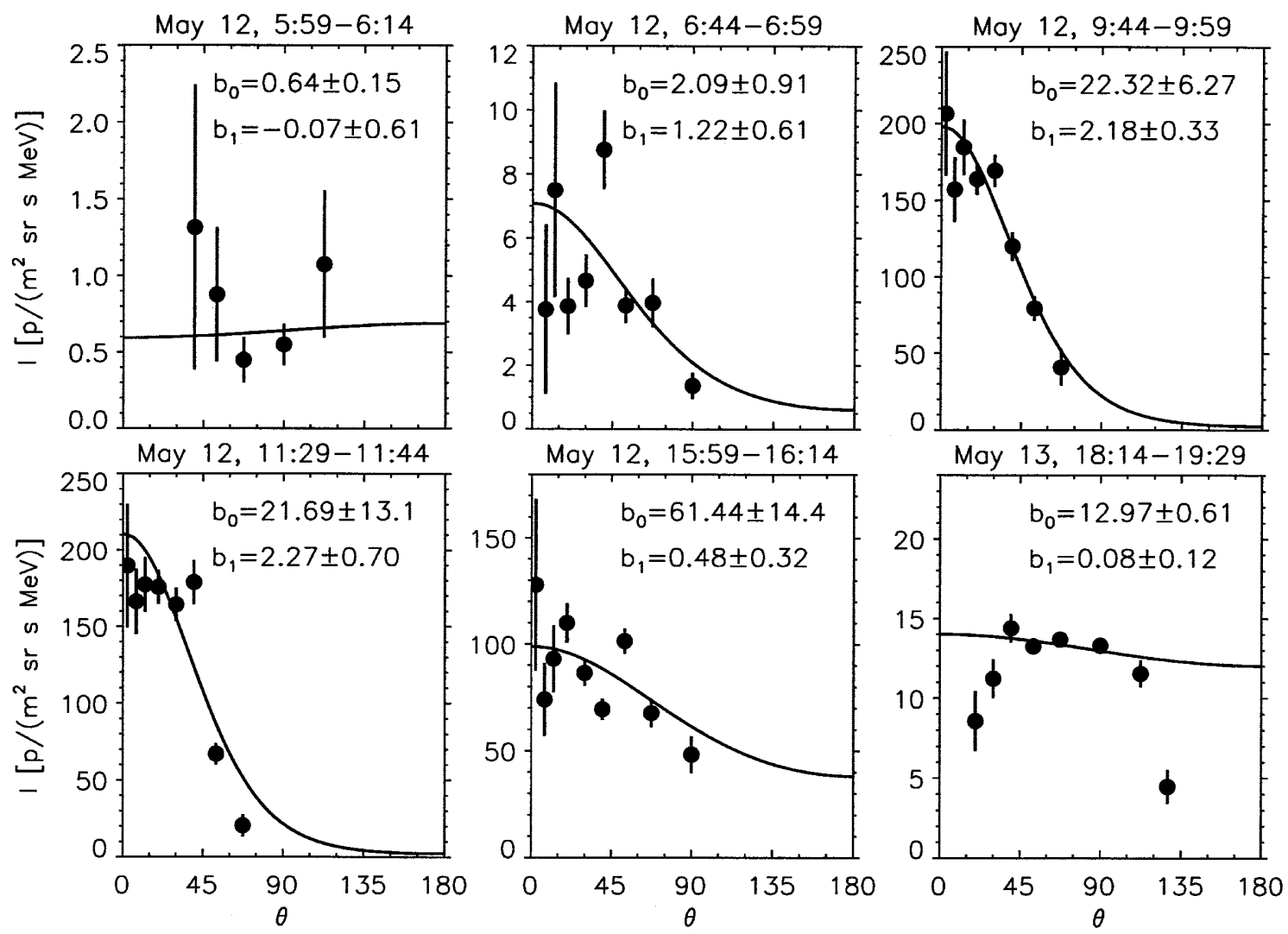

Fig. 2. Pitch angle distributions in six time intervals throughout the May 12 event. The time interval in question is indicated above each frame. Fits to Eq. 1 are shown as solid lines. The error bars show the statistical $1 \sigma$ error limits. Values for fitting parameters are shown within the frames

Ramaty, 1985) for the proton source function of the injection:

$N(E, t)=N_{0} \frac{\left(\varepsilon+\varepsilon_{m}\right)}{\left(2 \varepsilon_{m} \varepsilon+\varepsilon^{2}\right)^{\frac{\gamma(t)+1}{2}}} \exp \left(-\frac{E}{E_{0}}\right)$,

where $E$ is the particle energy, $m$ is the proton mass, $\varepsilon=E / E_{1}, \quad \varepsilon_{m}=m c^{2} / E_{1}, \quad$ and $\gamma(t)=(\sigma+2) /(\sigma-1)$ with $\sigma$, which in original model of Ellison and Ramaty (1985) is compression ratio of the shock, being a fitting parameter depending linearly on time

$\sigma=\sigma_{0}+\sigma_{1}\left(t-t_{1}\right), \quad$ when $t>t_{1}$ and $\sigma_{0}+\sigma_{1}\left(t-t_{1}\right)>1$

$\sigma \rightarrow 1$, when $t<t_{1}$ or $\sigma_{0}+\sigma_{1}\left(t-t_{1}\right) \leq 1$,

where $\sigma_{0}, \sigma_{1}$ and $t_{1}$ are fitting parameters. The injection energy $E_{1}$ was selected to be $0.5 \mathrm{MeV}$ (see Anttila et al., 1998). The cut-off energy $E_{0}$ and normalization factor $N_{0}$ are fitting parameters.

As shown in a multi-spacecraft study by Kallenrode (1996), the shock acceleration efficiency seems to decrease roughly symmetrically with increasing longitudinal distance from the shock nose. Therefore, the full injection function is of the form

$q(E, t)=N(E, t) \exp \left(-g_{1}\left|\varphi-\varphi_{0} \frac{t}{t_{s}}\right|\right)$,

where $N(E, t)$ is the spectrum given in Eq. (2), $\varphi$ is the heliolongitudinal distance of the event associated flare from the solar footpoint of the IMF line connected to $\mathrm{SOHO}, \varphi_{0}$ is the heliolongitude of the footpoint of the IMF line connected to SOHO, and $t_{s}$ is the shock transit time calculated from the event onset at the Sun. Lario et al. (1998) found in their multi-spacecraft study of proton acceleration in IP shocks that this longitudinal effect should be energy dependent. However, it is not possible to fully separate radial and longitudinal effects in a study made from observations of a single spacecraft. Therefore, we chose a simplified model, where the energy dependence is involved with radial effect (in fact the radial evolution of injection rate is parametrized as a temporal evolution in Eq. 2). The flare site is selected to represent the initial position of the shock nose at the onset of the event. Sometimes centre of a CME and associated flare can be apart by as much as $180^{\circ}$ from each other, but for these events the assumption is justified, because in both cases there was a halo CME detected by LASCO (LASCO CME list, http://lascowww.nrl.navy.mil $/ \mathrm{cmelist.html}$ ) and a flare in the central part of the solar disk (Solar-Geophysical Data, SGD). It should also be noticed that the shock nose represents the site of the strongest acceleration, and is not necessarily situated in the centre of the CME. During the two events under study, the longitudinal distance from the shock nose had little meaning on our results as will be shown. The distance of the shock from spacecraft along the IMF field lines is assumed to decrease linearly with time. 
This assumption means that the shock is slowly decelerating during its passage through the IP space, which is in accordance with observations. CMEs faster than the ambient solar wind are decelerating, and CMEs slower than the solar wind are accelerating during their passage through the heliosphere (e.g., Gosling, 1996).

The IP transport of the particles is treated numerically by using Monte Carlo simulations of Green's functions as described by Torsti et al. (1996). The interplanetary transport is calculated from the pitch angle distribution integrated over the whole event. The parallel mean free path (MFP), $\lambda_{\|}$is obtained from

$\lambda_{\|}=b_{1} \mathrm{~L}$,

where $\mathrm{L}$ is the focusing length. On the other hand

$\lambda_{\|}=\lambda_{\mathrm{r}} \sec ^{2}(\psi)$,

where $\lambda_{\mathrm{r}}$ is radial MFP and $\psi$ is the angle between the radial direction and the IMF. We used the rigidity dependence of $\lambda_{\mathrm{r}} \sim \mathrm{P}^{1 / 3}$, which results from the standard quasi-linear theory with the Kolmogorov spectrum of turbulence (e.g., Kunow et al., 1991). We obtained values $\lambda_{\mathrm{r}}(1 \mathrm{GV})=0.37 \mathrm{AU}$ for the April 7 event and $\lambda_{\mathrm{r}}$ $(1 \mathrm{GV})=0.15 \mathrm{AU}$ for the May 12 event. The best fit for the observed intensity at $1 \mathrm{AU}$ was then obtained by convoluting the Monte Carlo simulated IP transport Green's function with the source function $q(E, t)$ (Torsti et al., 1996; Anttila et al., 1998).

\section{Observations}

The April 7, 1997 event was associated with a C6.8-class X-ray flare with a maximum at 14:03 UT at S30 E19, and type II, III and IV radio bursts (SGD). A halo CME was observed by $\mathrm{SOHO} / \mathrm{LASCO}$ at 14:27 UT with a speed of $\sim 700 \mathrm{~km} / \mathrm{s}$ simultaneously with a Moreton wave expansion detected by SOHO/EIT (Bothmer et al., 1997; Thompson et al., 1999). The energetic proton intensities in ERNE started to rise at 15:15 UT in the 24-48 MeV energy channel. At $23: 30$ UT (570 minutes in Fig. 3), the intensities in all energy channels dropped by $\sim 50 \%$. The passage of the interplanetary shock at the SOHO site was detected as an intensity increase in the ERNE 1.6-3 MeV proton channel and as an increase of the solar wind speed from $\sim 300 \mathrm{~km} / \mathrm{s}$ to $\sim 450 \mathrm{~km} / \mathrm{s}$ by CELIAS/MTOF proton monitor on April 10, 12:58 UT (http://umtof.umd.edu/pm). The energetic particle intensity profiles observed by ERNE are shown in Fig. 3.

Similar phenomena occurred during May 12, 1997 event. A C1.3-class X-ray flare was observed at N21 W09 with maximum at 4:52 UT. It was associated with type II, III and IV radio emissions (SGD), a halo CME observed by LASCO at 6:30 UT (http://lascowww.nrl.navy.mil/cmelist.html) and a Moreton wave observed by EIT (http://umbra.nascom.nasa.gov/eit/ cme/may12/index.html). The onset of the SEP event was detected in the ERNE 24-48 MeV proton channel at 6:05 UT. At the time of the interplanetary shock passage, on May 15, 0:58 UT, the solar wind velocity observed by CELIAS/MTOF increased from $\sim 300 \mathrm{~km} / \mathrm{s}$

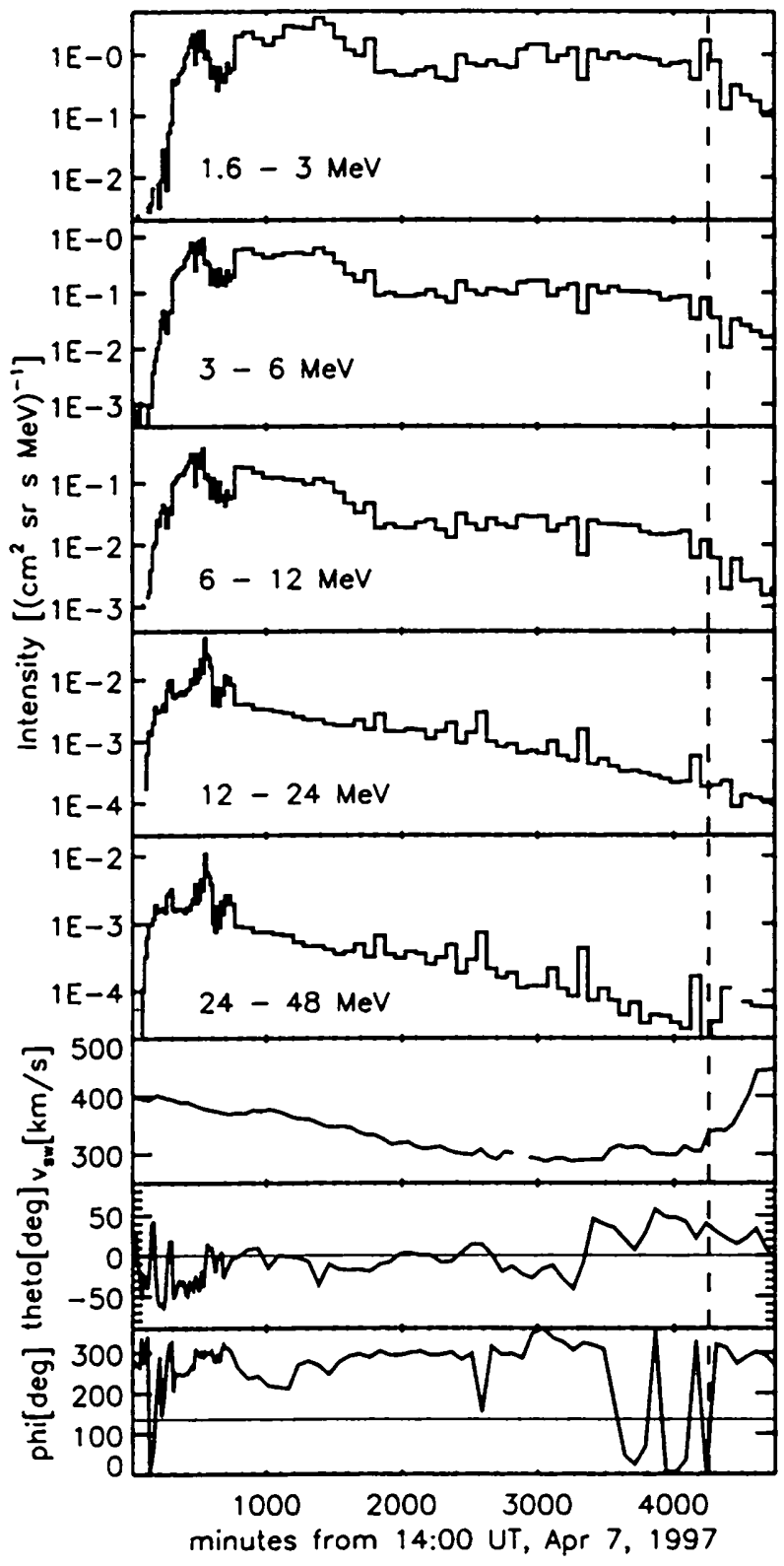

Fig. 3. Omnidirectional energetic proton intensities in energy channels $1.6-3,3-6,6-12,12-24$ and $24-48 \mathrm{MeV}$ (five uppermost panels) measured by ERNE during the April 7, 1997 Earth-directed CME. The data is 15-min averaged for the first $750 \mathrm{~min}$ from the event onset (14:00 UT, April 7, 1997), and 75-min averaged after $750 \mathrm{~min}$ from the event onset. One hour averaged solar wind velocity (sixth panel) from CELIAS/MTOF proton monitor (http://umtof.umd.edu/pm). IMF direction in GSE coordinates with $\theta$ (seventh panel) and $\varphi$ (eighth panel). The horizontal lines show nominal direction of ERNE. The passage of the shock is marked by a dashed vertical line

to $\sim 450 \mathrm{~km} / \mathrm{s}$ (http://umtof.umd.edu/pm), and the proton intensities in ERNE increased up to $>20 \mathrm{MeV}$ energies as seen from Fig. 4.

\section{Results and discussion}

We have fitted the first $3600 \mathrm{~min}$ from the onset of both of the events with a chi squared method in such a manner that, in order to give more importance for the rise phase of the events, during the first $750 \mathrm{~min}$ the data 


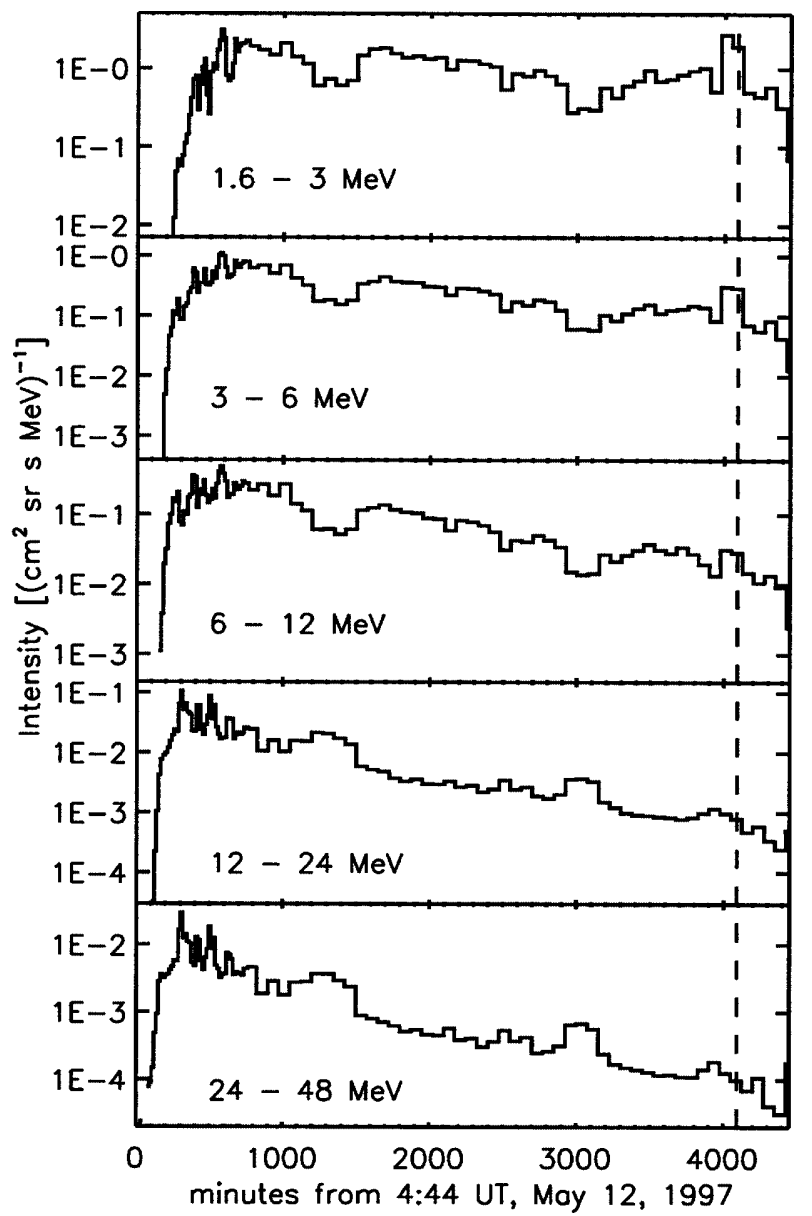

Fig. 4. Same as Fig. 3, but for the May 12, 1997 event. The onset time of the event is 4:44 UT, May 12, 1997

is 15 -min averaged and from 750 to $3600 \mathrm{~min} 75-\mathrm{min}$ averaged. The reason why the fitting was stopped at $3600 \mathrm{~min}$, is that our model with spatially constant MFP cannot fit the intensity increase during the shock passage (the shock passage times were 4258 and 4091 min for April 7 and May 12 events, respectively). The increase during the shock passage is due to the fact that close to the shock MFP is much shorter than elsewhere in the IP space, and only a minor portion of the particles can escape the region around the shock.

The fits of 1.6-48 MeV proton intensities for the events on April 7 and May 12 are shown in Figs. 5 and 6 , respectively. In the case of the event of April 7, the situation for ERNE anisotropy measurements is rather problematic. The magnetic field was situated $\sim 90^{\circ}$ from the ERNE nominal direction (Fig. 3), and fluctuated strongly. Therefore our model does not work perfectly for every moment of time, and the intensity profiles (Figs. 3, 5) also show fluctuations, especially in low energies. These anomalies can partially result from the fact that the anisotropy distribution is of a more complex form than assumed in Eq. 1. An additional problem was caused by a sudden intensity decrease of $\sim 50 \%$ in all energy channels at $\sim 23: 30$ UT on April 7 . The decrease was probably a result of the spacecraft entering a flux tube, where the IP transport conditions

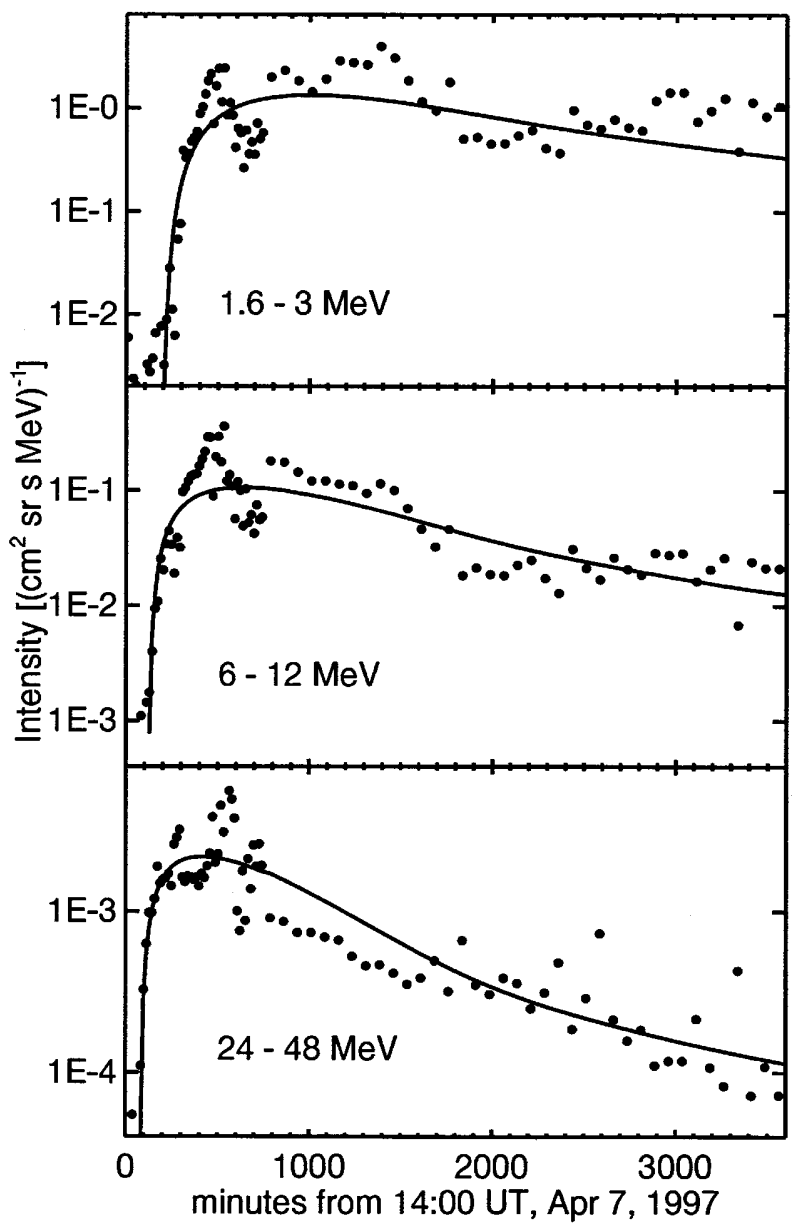

Fig. 5. Fit of our model (solid lines) for the April 7 event in proton energy channels 1.6-3, 6-12 and 24-48 MeV (Solid circles)

were different compared to flux tubes where the spacecraft was situated earlier. Because our model uses constant MFP, it cannot fit these kind of changes in IMF conditions, but the fitted curves are fits for averaged conditions during the whole event. However, we were able to fit our model reasonably well with the observations. In the case of May 12 event the situation was easier. The magnetic field was for most of the time inside the HED view cone, and fluctuations were smaller than during the April 7 event (Fig. 4). This can be seen as smoother intensity profiles in Figs. 4 and 6, where only a few "bumps" exist. In particular during the May 12 event, it can be seen that the fit in the $24-48 \mathrm{MeV}$ energy channel does not decay as fast as the observations. This is probably a sign of rigidity dependence of MFP being somewhat stronger than $\mathrm{P}^{1 / 3}$ used in our model.

The parameters of our model for the April 7 and May 12 events are compared with the Earth-directed CME on October 19, 1989 (Fig. 7), analyzed earlier by Anttila et al. (1998), in Table 1. It is noteworthy that the parameters by themselves do not tell us a lot about the nature of the events. Although we use the model of Ellison and Ramaty (1985), we do not claim that the observed intensities are necessarily created by first order Fermi acceleration in a shock with compression ratio $\sigma$, 


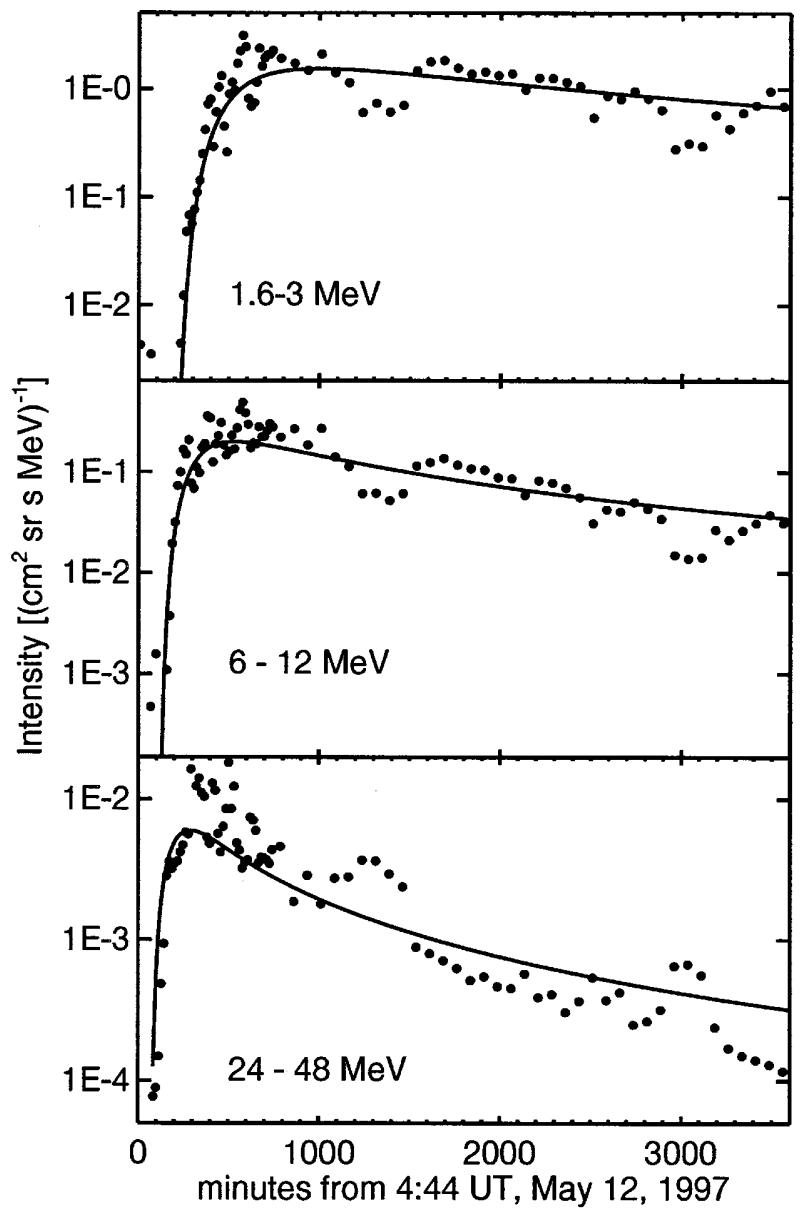

Fig. 6. Same as Fig. 5, but for the May 12 event

but our aim is to find a numerical solution for spectral form and temporal evolution of injection in the source. It is possible that other spectral forms can explain the observations as well. Therefore it is more important to compare the deconvoluted injection rates as in Fig. 8.

In comparison between the events under study and a much more intensive and faster CME with energetic particle intensities of several orders of magnitude higher (the event on October 19, 1989), the biggest difference is naturally in values of $N_{0}$, which gives the total number of the particles injected during the onset of the event. Although the absolute value of $\sigma_{1}$ is even larger during the October 19, 1989 event than during the May 12, 1997 event, we have to remember that $\sigma_{1}$ is a function of time, not of distance. The shock associated with the CME of the October 19, 1989 event reached 1 AU about three times is fast as the shocks of the year 1997 under study, and also the MFPs for the events in 1997 were longer than in the case of October 19, 1989 event. Therefore the most important difference between the intensive event during the solar maximum and the weaker events during solar minimum is that the particle injection rate from the shock decreases rapidly as can be seen from the Fig. 8.

Despite the rapid decrease of injection, the intensity profiles (Figs. 3, 4) show rather typical behaviour of Earth-directed CMEs with slow decay, when the shock

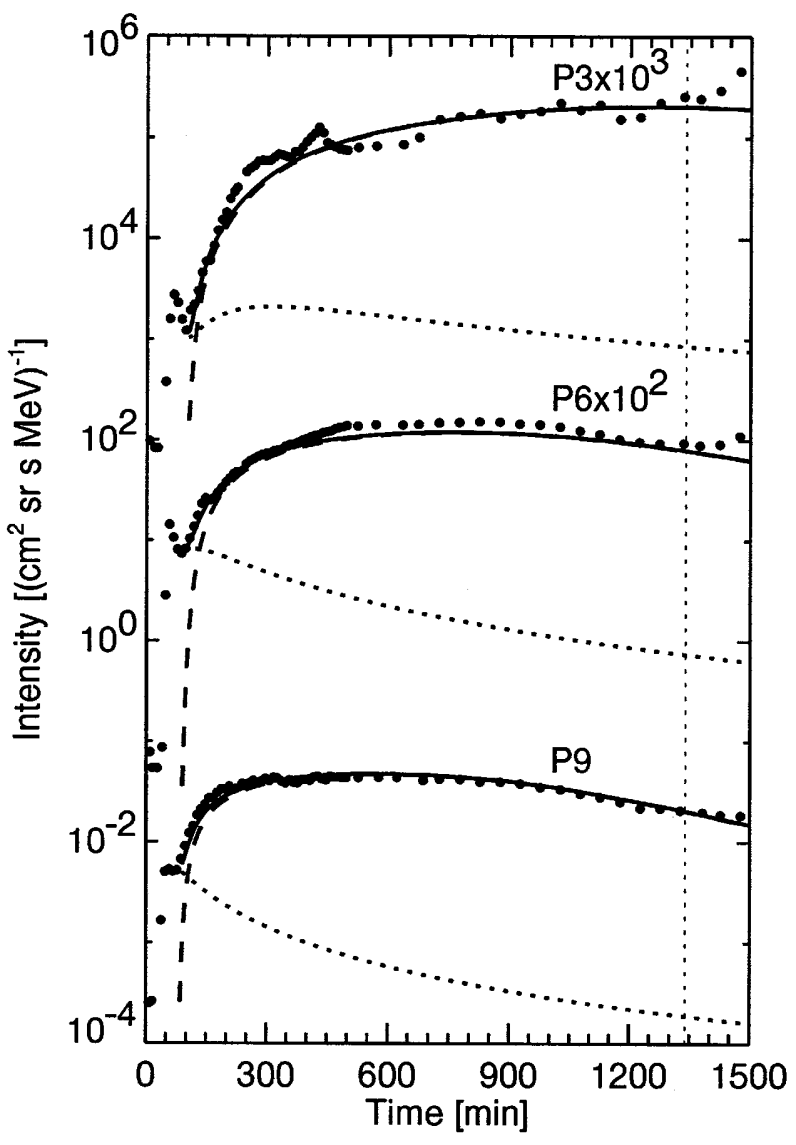

Fig. 7. Observed (GOES-6, Solid circles) and calculated pre-shock count rates of the October 19, 1989 SEP event in proton channels P3, P6 and P9 corresponding to the nominal energy ranges $8.7-14.5 \mathrm{MeV}$, 84-200 MeV, and 430-505 MeV, respectively (a detailed description of the secondary channels is given in Table A1 of Anttila et al., 1998). For the first $500 \mathrm{~min}$, the data is 10 -min averaged, and from 500 to $1500 \mathrm{~min} 50$-min averaged. The calculated prompt component is marked by the dotted line, the delayed component by the dashed line and their sum by the solid line. The vertical dotted line shows the shock passage (for description of the components, see Anttila et al., 1998). The figure has been reprinted from Anttila et al. (1998)

propagates to longer distances from the Sun. The intensities decrease somewhat faster than during the October 19, 1989 event (see Fig. 7), but an extreme difference can be seen in development of the injection rate. During the April 7 event, the particles injected, when the shock was at distances $<0.5 \mathrm{AU}$ from the

Table 1. Injection parameters of three Earth-directed CMEs on October 19, 1989, April 7, 1997, and May 12, 1997

\begin{tabular}{llll}
\hline & $\begin{array}{l}\text { October } \\
19,1989\end{array}$ & $\begin{array}{l}\text { April } \\
7,1997\end{array}$ & $\begin{array}{l}\text { May } \\
12,1997\end{array}$ \\
\hline$\lambda_{\mathrm{r}}(1 \mathrm{GV})[\mathrm{AU}]$ & 0.1 & 0.37 & 0.15 \\
$N_{0}\left[(\mathrm{~s} \mathrm{hemisphere} \mathrm{MeV})^{-1}\right]$ & $1.3 \cdot 10^{34}$ & $1.2 \cdot 10^{32}$ & $8.4 \cdot 10^{31}$ \\
$E_{0}[\mathrm{MeV}]$ & 235 & 15 & 15 \\
$t_{1}[\mathrm{~min}]$ & 60 & 35 & 10 \\
$\sigma_{0}$ & 3.4 & 2.8 & 2.95 \\
$\sigma_{1}\left[\mathrm{~min}^{-1}\right]$ & -0.00075 & -0.0004 & -0.0022 \\
$g_{1}\left[\mathrm{deg}^{-1}\right]$ & 0.066 & 0.043 & 0.028 \\
\hline
\end{tabular}




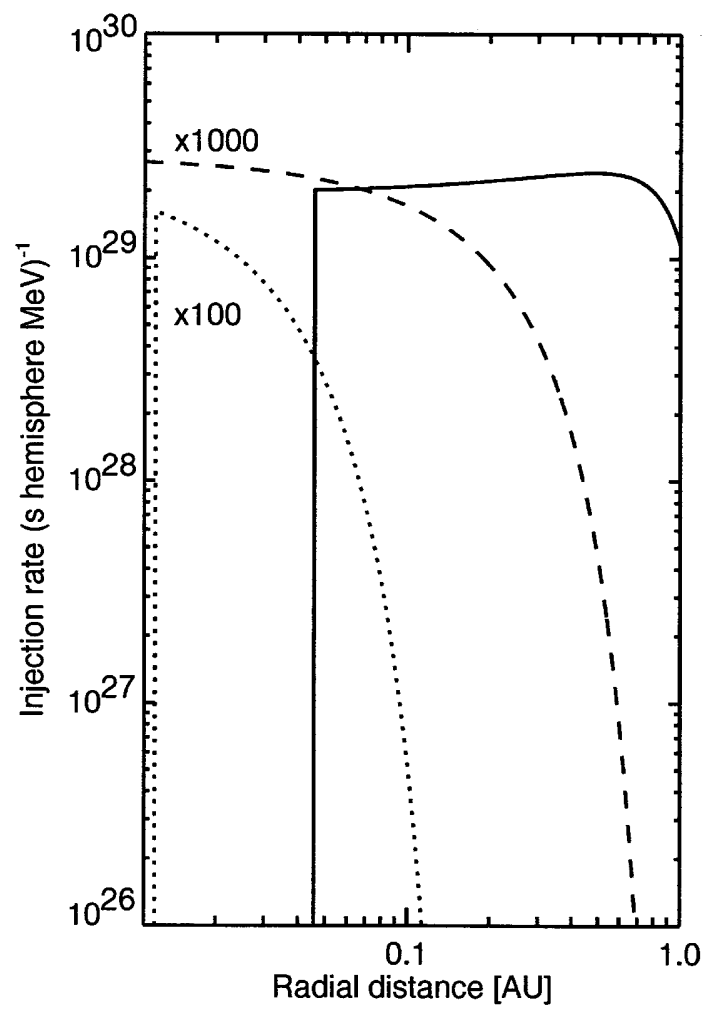

Fig. 8. Comparison of the injection rate of $10 \mathrm{MeV}$ protons between the October 19, 1989 (solid line), April 7, 1997 (dashed line) and May 12, 1997 (dotted line) CMEs. In order to show all of the events in the same scale, injection rates of April 7 and May 12 events are multiplied by 1000 and 100 , respectively. Notice that at the distances $<0.5 \mathrm{AU}$ the injection rate of the October 19, 1989 event is still increasing, while during the two other events, the injection practically vanishes during the shock propagation to $0.5 \mathrm{AU}$

Sun, dominated the flux till the passage of the shock (Figs. 8, 9). In the case of May 12 event, the injection rate decreased faster, and became meaningless already at the distance of $\sim 0.1$ AU (Figs. 8, 10). During the October 19, 1989 event the injection rate increased, when the shock propagated from the Sun to $\sim 0.5 \mathrm{AU}$, and the decay after the maximum was slow. The number of $10 \mathrm{MeV}$ protons injected, when the shock was less than 0.1 AU from the Sun, was only $\sim 5 \%$ of the number of $10 \mathrm{MeV}$ protons injected, when the shock was between 0.1 and $1 \mathrm{AU}$. During the April 7 event these populations were roughly equal, and during the May 12 event, the injection farther away from $0.1 \mathrm{AU}$ was meaningless. The shock speed at $1 \mathrm{AU}$ was $\sim 450 \mathrm{~km} / \mathrm{s}$ in both cases, and thus this result is in accordance with the study of Reames et al. (1997), in which they examined $1.15-63.2 \mathrm{MeV}$ protons during 56 CME associated events, and remarked that shocks with transit speeds below $500 \mathrm{~km} / \mathrm{s}$ had no SEP association.

This kind of early decay of injection makes the parameter $g_{1}$ rather meaningless, but for uniformity we did not drop it from our model, although it would have been possible. We also consider the fact that in eastern and central meridian (like the events under study) CMEs, the parameters $\sigma_{1}$ and $g_{1}$ are not independent of each other, because the observations by a single

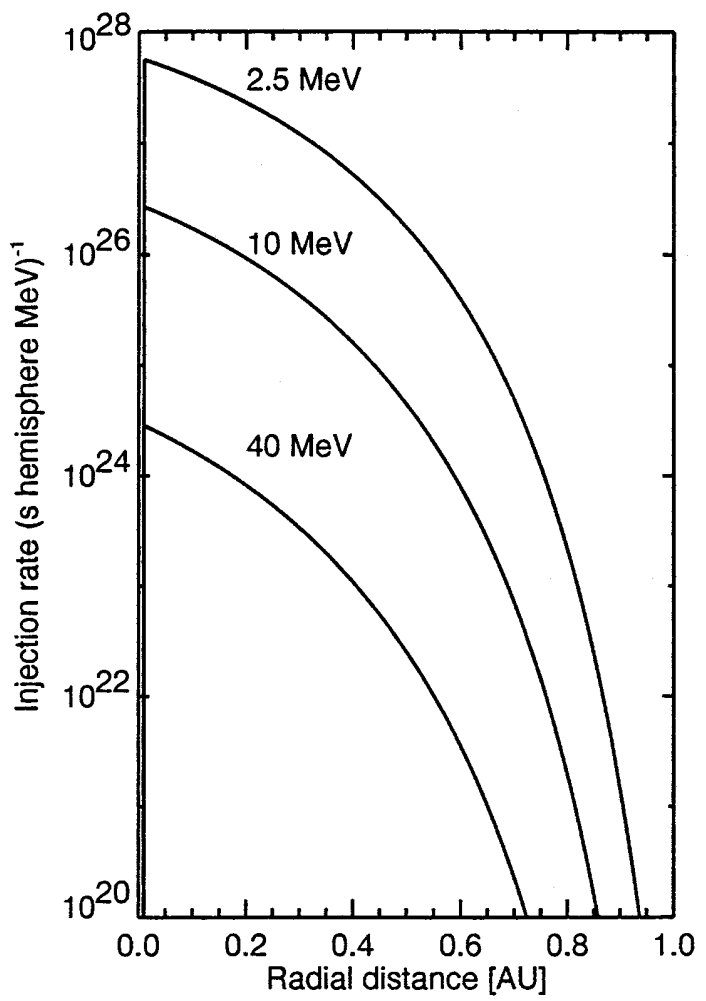

Fig. 9. Deconvoluted injection profile for $2.5,10$ and $40 \mathrm{MeV}$ protons during the April 7 event. Notice that injection rate became rather meaningless, when the shock was $\sim 0.5$ AU from the Sun

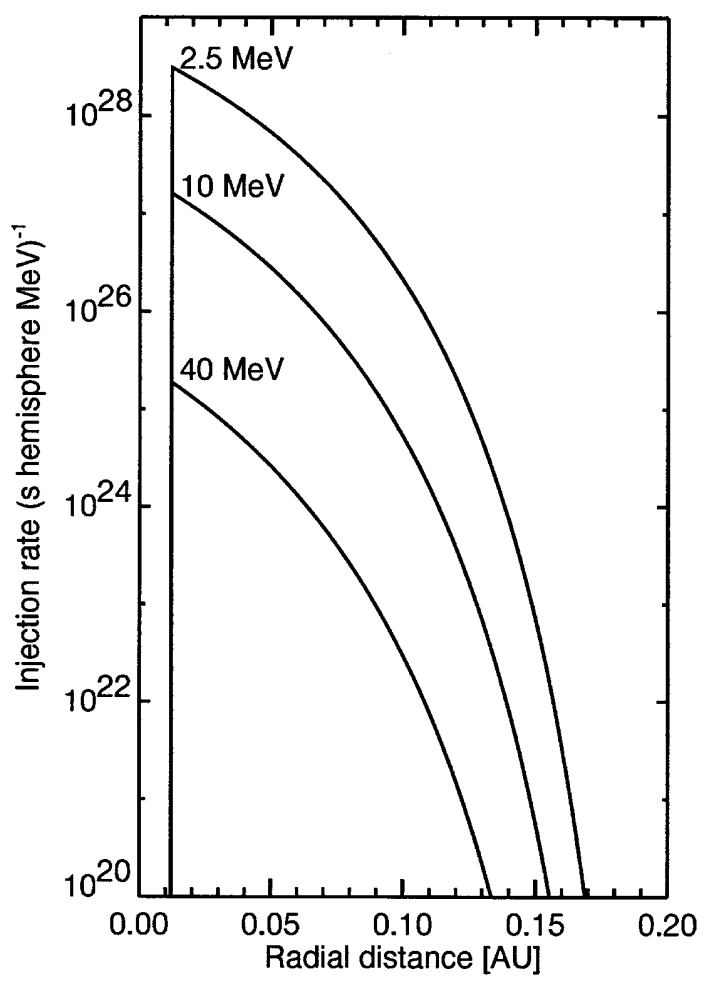

Fig. 10. Same as Fig. 9, but for the May 12 event. In this case the injection rate decreased by several orders of magnitude at the distances $<0.1 \mathrm{AU}$ from the Sun 
spacecraft do not allow us to distinguish between the effect of weakening of the shock as a function of the distance from the Sun (Lario et al., 1998) and the increase of the acceleration efficiency, when the footpoint of the IMF line connected to spacecraft approaches the shock nose (Kallenrode, 1996).

The comparison between the values of $t_{1}$ does not give much information about the characteristics of the events, because in the case of April 7 and May 12, 1997 events, the statistics does not allow us to make firm conclusions of the onset times. During the October 19, 1989 event, there were clear signs of two-component structure, and the delayed component was injected $\sim 60$ min later than the prompt component (Anttila et al., 1998). During the April 7, 1997 event there were possibly also signs of two components, but the statistics of ERNE data does not allow us be sure about that. On May 12, 1997, a precursor was clearly seen in the $24-48 \mathrm{MeV}$ proton channel of ERNE (Torsti et al., 1998). Even if both of the April 7 and May 12 events consisted of two components, the value of $t_{1}$ does not reveal the temporal difference of onset times of the two components, as was the case in October 19 event. The statistics for these less intensive events are simply too inconclusive.

The injection spectra integrated over the events of April 7 and May 12 can be seen in Fig. 11. Despite the different kind of time profiles of the injection, the timeintegrated spectra are nearly identical. The October 19, 1989, event is not shown in the figure, because the injection rate at the time of the shock passage was still remarkably high ( $\sim 20 \%$ of its maximum value), and our model can not fit the injection from the outer heliosphere. Thus integration over the whole event is impossible in the case of October 19, 1989 CME. The similarity of integrated injection spectra between April 7 and May 12 event raises a question whether the injection profiles really differ that much from each other. As mentioned, the strongly fluctuating IMF was problematic during the April 7 event, and may have falsified our determination of MFP. Therefore we attempted to fit the April 7 event with the same MFP as May 12 event, and the injection profiles were naturally rather similar, with the April 7 event decaying even slightly faster than the May 12 event. However, that does not affect the fact that the injection from these shocks decayed much faster than was the case with the October 19, 1989 CME.

However, around the shock passage an increase of intensities (seen especially during the May 12 event) was observed. This reveals that the shock was still accelerating and/or storing particles during its propagation into the outer heliosphere, but due to the extreme short MFP close to the shock, only a minor amount of these particles could escape the region nearby the shock. The particles generate resonant waves, which form this wavetrapping region, where the intensities of particles and waves depend only on distance from the shock. These energetic storm particles are seen up to $\mathrm{MeV}$ or tens of MeVs energies (Lee, 1997 and references therein). Our model with spatially constant MFP can not fit that particle population, but only simulates the particles injected from the "short MFP region" around the

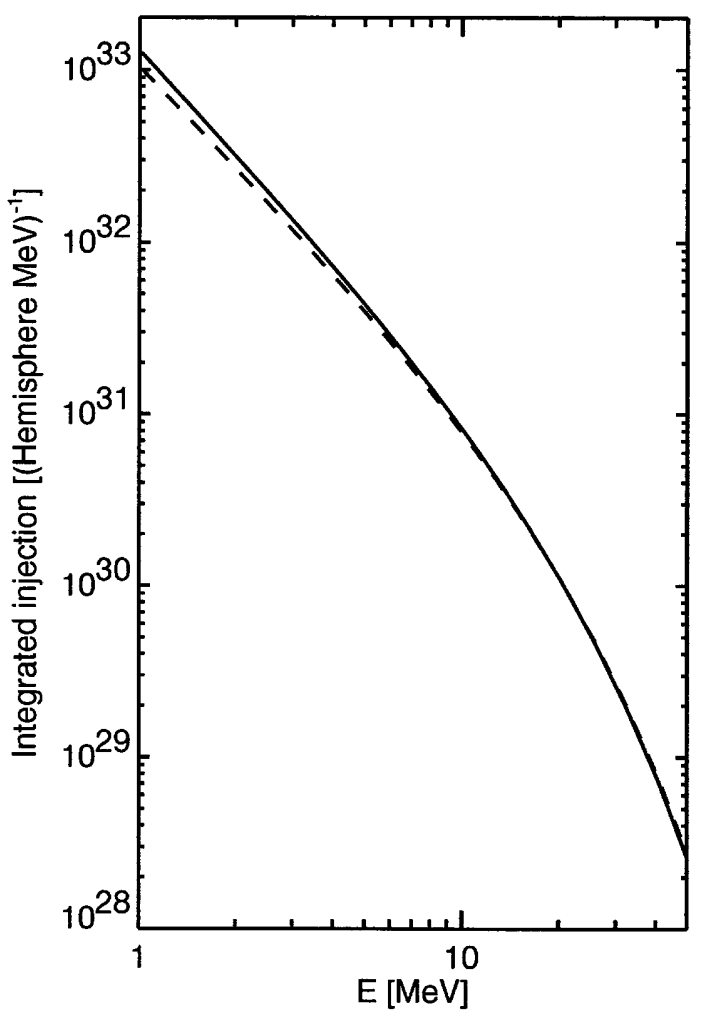

Fig. 11. Injection spectra of April 7 (solid line) and May 12 (dashed line) events integrated over the whole event

shock, and that injection had died out at the time of the shock passage.

\section{Conclusions}

We have developed a method for estimation of omnidirectional energetic particle intensities from the ERNE intensity and anisotropy observations. The method gives reasonable results especially in cases, when the fluctuations of IMF direction are small and transport conditions in the IP space are stable. The Earth-directed CME events on April 7 and May 12, 1997 have been analyzed by this method.

The results for $1.6-48 \mathrm{MeV}$ protons show that, compared to a much more intensive and faster $\mathrm{CME}$ during the previous solar maximum (the event on October 19, 1989), the injection rate of $>1 \mathrm{MeV}$ protons from the shock of April 7 CME decreased fast, and the injection became meaningless at distances $<0.5 \mathrm{AU}$ from the Sun. In the case of May 12 event, the injection rate had decreased by several orders of magnitude, when the shock was only 0.1 AU from the Sun.

The shock was still accelerating and/or storing particles during its propagation to outer heliosphere, but due to a short MFP close to the shock only a minor portion of these particles could escape the region close to the shock.

Acknowledgements. We thank Rami Vainio for the calculation of numerical Green's functions and fruitful discussions of the 
phenomena associated with energetic particle acceleration and transport processes. We are grateful to Jean-Pierre Delaboudinière for the permission to use SOHO/EIT data, to Chris St. Cyr for the use of $\mathrm{SOHO} / \mathrm{LASCO}$ data, to Fred M. Ipavich for the use of SOHO/CELIAS/MTOF data, and to Ron Lepping for the use of WIND/MFI data. The Academy of Finland is thanked for financial support. SOHO is an international cooperation project between ESA and NASA.

Topical Editor E. Antonucci thanks V. Bothmer for his help in evaluating this paper.

\section{References}

Anttila, A., L. G. Kocharov, J. Torsti, and R. Vainio, Long-duration high-energy proton events observed by GOES in October 1989, Ann. Geophysicae, 16, 921, 1998.

Beeck, J., and G. Wibberenz, Pitch angle distributions of solar energetic particles and the local scattering properties of the interplanetary medium, Astrophys. J., 311, 437, 1986.

Boberg, P., A. Tylka, and J. Adams, Solar energetic Fe charge state measurements: implications for acceleration by coronal mass ejection-driven shock, Astrophys. J., 471, L65, 1996.

Bothmer, V., A. Posner, H. Kunow, R. Müller-Mellin, B. Heber, M. Pick, B. J. Thompson, J.-P. Delabodinière, G. E. Brueckner, R. A. Howard, D.-J. Michels, C. St. Cyr, A. Szabo, H. S. Hudson, G. Mann, H.-T. Classen, and S. McKenna-Lawlor, Solar energetic particle events and coronal mass ejections: new insights from SOHO, Proc of 31st ESLAB Symp., ESA SP415, 207, Noorwijk, 1997.

Ellison, D. C., and R. Ramaty, Shock acceleration of electrons and ions in solar flares, Astrophys. J., 298, 400, 1985.

Gosling, J. T., The solar flare myth, J. Geophys. Res., 98, 18 937, 1993.

Gosling, J. T., Corotating and transient solar wind flows in three dimensions, Annu. Rev. Astron. Astrophys., 34, 35, 1996.

Gosling, J. T., Coronal mass ejections: an overview, in Coronal mass ejections, Eds. N. Crooker, J. A. Joselyn, and J. Feynman, AGU, Washington D.C., p. 9, 1997.

Kallenrode, M.-B., A statistical survey of $5-\mathrm{MeV}$ proton events at transient interplanetary shocks, J. Geophys. Res., 101, 24 393, 1996.

Klein, K.-L., E. L. Chupp, G. Trottet, A. Magun, P. P. Dunphy, E. Rieger, and S. Urpo, Flare-associated energetic particles in the corona and at 1 AU, Astron. Astrophys., 348, 271, 1999.
Kunow, H., G. Wibberenz, G. Green, R. Müller-Mellin, and M.-B. Kallenrode, in Physics of the inner heliosphere II, Eds. R. Schwenn, and E. Marsch, Springer, Berlin Heidelberg New York, 243, 1991.

Lario, D., B. Sanahuja, and A. M. Heras, Energetic particle events: efficiency of interplanetary shocks as $50 \mathrm{keV}<\mathrm{E}<100 \mathrm{MeV}$ proton accelerators, Astrophys. J., 509, 415, 1998.

Lee, M. A., Particle acceleration and transport at CME-driven shocks, in Coronal mass ejections, Eds. N. Crooker, J. A. Joselyn, and J. Feynman, AGU, Washington D.C., 227, 1997.

Lim, T. L., J. J. Quenby, M. K. Reuss, E. Keppler, H. Kunow, B. Heber, and R. J. Forsyth, Can diffusive shock acceleration work fast enough in interplanetary shocks? Evidence from the November 1992 event, Proc. of 24th ICRC (Rome), 4, 353, 1995.

Lumme, M., Data analysis of the energetic particle experiment onboard the SOHO satellite, PhD Thesis, Painosalama Oy, Turku, Finland, 1995.

Reames, D. V., S. W. Kahler, and C. K. Ng, Spatial and temporal invariance in the spectra of energetic particles in gradual solar events, Astrophys. J., 491, 414, 1997.

Tan, L. C., G. M. Mason, B. Klecker, and D. Hovestadt, Seed population for $\sim 1 \mathrm{MeV}$ per nucleon heavy ions accelerated by interplanetary shocks, Astrophys. J., 345, 572, 1989.

Thompson, B. J., J. B. Gurman, W. M. Neupert, J. S. Newmark, J.-P. Delaboudinière, O. C. St. Cyr, S. Stezelberger, K.-P. Dere, R. A. Howard, and D. J. Michels, SOHO/EIT observations of the 1997 April 7 coronal transient: possible evidence of coronal Moreton waves, Astrophys. J., 517, L151, 1999.

Torsti, J., E. Valtonen, M. Lumme, P. Peltonen, T. Eronen, M. Louhola, E. Riihonen, G. Schultz, M. Teittinen, K. Ahola, C. Holmlund, V. Kelhä, K. Leppälä, P. Ruuska, and E. Strömmer, Energetic particle experiment ERNE, Sol. Phys., 162, 505, 1995.

Torsti, J., L. G. Kocharov, R. Vainio, A. Anttila, and G. A. Kovaltsov, The 1990 May 24 solar cosmic ray event, Sol. Phys., 166, 135, 1996.

Torsti, J., A. Anttila, L. Kocharov, P. Mäkelä, E. Riihonen, T. Sahla, M. Teittinen, E. Valtonen, T. Laitinen, and R. Vainio, Energetic $(\sim 1$ to $50 \mathrm{MeV})$ protons associated with Earth-directed coronal mass ejections, Geophys. Res. Lett., 25, 2525, 1998.

Vainio, R., Monte-Carlo simulations of interplanetary transport and acceleration of energetic particles, PhD Thesis, Painosalama Oy, Turku, Finland, 1998. 